Canadian

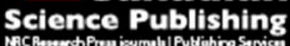

Applied Physiology, Nutrition, and Metabolism Physiologie appliquée, nutrition et métabolisme

\title{
Influence of sex on performance fatigability of the plantar flexors following repeated maximal dynamic shortening contractions
}

\begin{tabular}{|r|l|}
\hline Journal: & Applied Physiology, Nutrition, and Metabolism \\
\hline Manuscript ID & apnm-2017-0013.R3 \\
\hline Manuscript Type: & Brief communication \\
\hline Date Submitted by the Author: & O1-Jun-2017 \\
\hline Complete List of Authors: & $\begin{array}{l}\text { Lanning, Amelia; University of Oregon, Human Physiology } \\
\text { Power, Geoffrey; University of Guelph, Human Health \& Nutritional } \\
\text { Sciences } \\
\text { Christie, Anita; University of Oregon, Human Physiology } \\
\text { Dalton, Brian; University of British Columbia Okanagan, School of Health } \\
\text { and Exercise Sciences }\end{array}$ \\
\hline $\begin{array}{r}\text { Is the invited manuscript for } \\
\text { consideration in a Special } \\
\text { Issue? : }\end{array}$ & \\
\hline Keyword: & Sex, Velocity, Electromyography, muscle fatigue < muscle, power output \\
\hline &
\end{tabular}


Influence of sex on performance fatigability of the plantar flexors following repeated maximal dynamic shortening contractions

Amelia C Lanning ${ }^{1}$, Geoffrey A Power ${ }^{2}$, Anita D Christie ${ }^{1}$, Brian H Dalton ${ }^{3}$

${ }^{1}$ Department of Human Physiology, University of Oregon, Eugene, Oregon, United States

${ }^{2}$ Department of Human Health and Nutritional Sciences, University of Guelph, Guelph, Canada

${ }^{3}$ School of Health and Exercise Sciences, The University of British Columbia, Kelowna, Canada

\section{Brief Communication}

Running Title: Sex-related fatigability

Correspondence: Brian H Dalton, $\mathrm{PhD}$

School of Health and Exercise Sciences

The University of British Columbia

ART 360, 1147 Research Road

Kelowna, BC, Canada, V1V 1V7

E-mail: brian.dalton@ubc.ca

Telephone: 1-250-807-9513 


\begin{abstract}
The purpose was to determine sex-differences in fatigability during maximal, unconstrained velocity, shortening plantar flexions. The role of time-dependent measures (i.e., rate of torque development (RTD), rate of velocity development (RVD), rate of neuromuscular activation) in such sex-related differences was also examined. By task termination, females exhibited less reductions in power and similar changes in rate of neuromuscular activation than males, indicating females were less fatigable than males.
\end{abstract}

Key Words: Sex, Velocity, Electromyography, Muscle Fatigue, Power Output 


\section{INTRODUCTION}

During isometric tasks performed at the same relative intensity, females are more fatigue resistant than males, but this generalization depends on the intensity and muscle group (Hunter 2016). During dynamic tasks, however, the evidence is less clear as to whether females exhibit less fatigability than males, which is likely owing to an inadequate number of studies focusing on sex-related fatigability and dynamic tasks (Hunter 2016).

Most studies focusing on isotonic-like tasks have focused on the elbow flexors and/or knee extensors (Maughan et al. 1986, Pincivero et al. 2004, Labarbera et al. 2013, Senefeld et al. 2013, 2016, Yoon et al. 2015) and have reported less (Maughan et al. 1986, Labarbera et al. 2013, Yoon et al. 2015) or similar (Maughan et al. 1986, Pincivero et al. 2004, Senefeld et al. 2013, 2016) fatigability for females compared with males. During controlled, slow velocity, low resistance contractions, females exhibited less fatigue than males (Labarbera et al. 2013; Maughan et al. 1986; Yoon et al. 2015), but as the load (Maughan et al. 1986) or angular velocity is increased (Senefeld et al. 2013) sex-related fatigability is similar. However, when fatigability was characterized by a maximal voluntary isometric contraction (MVC) following maximal unconstrained velocity knee extensions, females were less fatigable than males (Senefeld et al. 2013). Thus, owing to differences in task intensity (i.e., load), muscle group, and criterion measure, a decisive conclusion on sex-based differences in fatigue is unclear.

Maximal unconstrained velocity during isotonic contractions has become an informative parameter for characterization of the fatigue-related decrements in peak power for both young (Cheng and Rice 2005, Power et al. 2010, Senefeld et al. 2013) and older (Dalton et al. 2010, 2012, 2015, Power et al. 2012, Senefeld et al. 2016) adults. Time-dependent performance 
measures (i.e., rate of neuromuscular activation, rate of torque development (RTD), and rate of velocity development (RVD)) may provide key factors that fundamentally determine peak velocity and dynamic contraction performance (i.e., peak power) (Andersen and Aagaard 2006, Wallace et al. 2016). Following a fatiguing task, if there is indeed a sex-difference in power, these time-dependent measures may provide further insight into factors of sex-related performance fatigability.

The purpose of the present study was to determine whether females are less fatigable than males following an unconstrained velocity, shortening plantar flexion task and whether timedependent neuromuscular factors (i.e., RTD, RVD, and rate of neuromuscular activation) can explain the sex-related differences in performance fatigability. We hypothesized that following the fatiguing task, females would display less fatigue than males, as measured by less of a decrease in peak power, and fatigue-related changes in peak power will be related to RTD and RVD. 


\section{MATERIALS AND METHODS}

Participants. Nine females and eight males were recruited from the local university community. All participants were recreationally active (Table 1) and reported no lower limb neuromuscular or orthopedic disorders. Participants granted written informed consent. The procedures were approved by the University's institutional review board and conformed to the Declaration of Helsinki.

Experimental Arrangement. Plantar flexion torque, ankle position and angular velocity were recorded in isometric and isotonic modes of a Biodex System 3 (Biodex Medical Systems Inc., Shirley, NY, USA). Participants sat upright with the knee extended to $170^{\circ}$ (full extension $=$ $\left.180^{\circ}\right)$, hip joint at $85^{\circ}$, and ankle in a neutral position $\left(0^{\circ}\right.$ of plantar flexion $)$ for the isometric and initial position for the isotonic contractions (Dalton et al. 2013, 2014). The range of motion for the fatigue task was set from neutral to $25^{\circ}$ of plantar flexion. A custom-made binding and an inelastic strap over the dorsum and toes, respectively, secured the foot to the footplate.

All contractions were performed with the right plantar flexors and the ankle joint aligned with the dynamometer's axis of rotation. Torque, angular velocity, and position were sampled at 1000Hz (Power 1401-3, Cambridge Electronics Design, Cambridge, UK) and stored using Spike2 software (v7, Cambridge Electronics Design, Cambridge, UK).

In accordance with a previous report (Wallace et al. 2016), surface electromyography (EMG) signals were collected from the soleus and medial gastrocnemius (MG) using pairs of self-adhering electrodes (M-00-S, Ambu BlueSensor M, Ambu A/S, Ballerup, Denmark) and a ground over the right patella. Electromyogram signals were pre-amplified $(\times 100)$, amplified 
$(\times 2)$, filtered $(30-1000 \mathrm{~Hz})$, and sampled at $2000 \mathrm{~Hz}$ (Power 1401-3, Cambridge Electronics Design, Cambridge, UK).

Experimental Procedures. Participants performed 3-4 7-s MVCs with 3 min rest between efforts. An additional MVC was attempted if the first 3 MVCs varied in peak torque by more than $5 \%$.

Following 3 min of rest, participants were familiarized with the isotonic contractions by performing maximal plantar flexions through a $25^{\circ}$ range of motion (foot was passively returned to starting position) with a set resistance equivalent to $30 \%$ MVC. Participants attempted several contractions until a consistent peak angular velocity was achieved. Then, participants performed five consecutive contractions to practice the fatigue task. To ensure a maximal effort, each participant was instructed to plantar flex as quickly and forcefully as possible for all contractions, encouraged verbally, and given real-time visual feedback (Power et al. 2011).

Following 3-min rest, participants performed the fatigue task of 200 maximal-effort, isotonic plantar flexions with a 30\% MVC resistance. Participants contracted upon the "go" prompt of the investigator for a consistent frequency of 1 contraction per $\sim 1.5 \mathrm{~s}$. Participants attempted a final MVC $\sim 10 \mathrm{~s}$ following task termination.

Data and Statistical Analyses. Spike2 software (version 7, Cambridge Electronics Design, Cambridge, UK) was used to analyze all data. Torque and angular velocity channels were filtered digitally with a low-pass fourth-order digital filter at $40 \mathrm{~Hz}$. The baseline MVC with the highest peak torque was chosen to represent MVC peak torque (Nm). Peak power was calculated from the product of torque $(\mathrm{Nm})$ and velocity $(\mathrm{rad} / \mathrm{s})$. Dynamic voluntary RTD 
( $\Delta$ Torque/ $\Delta$ Time $)$ and RVD $(\Delta$ Velocity/ $\Delta$ Time $)$ were calculated using the same parameters as reported previously (Wallace et al. 2016). For rate of neuromuscular activation of the soleus $\left(\mathrm{RA}_{\mathrm{sol}}\right)$ and $\mathrm{MG}\left(\mathrm{RA}_{\mathrm{mg}}\right)$, rate of EMG rise was calculated (Wallace et al. 2016). Briefly, plantar flexor surface EMG was band-pass filtered digitally ( $4^{\text {th }}$ Order Butterworth, $\left.30-500 \mathrm{~Hz}\right)$ and the root mean square (RMS) amplitude was derived over a $50 \mathrm{~ms}$ iterative moving average window. Next, the linear slope ( $\Delta \mathrm{RMS}$ amplitude/ $\Delta$ Time) was determined using an iterative moving average window of $50 \mathrm{~ms}$ and the maximum value of the slope occurring between activation onset and prior to peak power was recorded for analysis. For analysis of all data, we defined baseline (i.e., pre-fatigue) as the maximum value within the first 10 contractions of the fatigue task; whereas the post-fatigue value was the average of the last 10 contractions.

Two-tailed, unpaired t-tests were used to compare baseline dependent variables between sexes. Further, the percent change in each variable was calculated ([Baseline - Post $]$ /Baseline) and compared between sexes with unpaired t-tests. Significance was set at $p<0.05$. Effect sizes were calculated using Cohen's $d$. Linear regression analyses $\left(r\right.$ and $\left.\mathrm{R}^{2}\right)$ were conducted to determine the relationship between fatigue-related change in peak power and changes in torque and velocity at peak power, $\mathrm{RTD}, \mathrm{RVD}, \mathrm{RA}_{\mathrm{sol}}$ and $\mathrm{RA} \mathrm{A}_{\mathrm{mg}}$. Data are reported as means \pm standard deviations. 


\section{RESULTS}

Baseline. Although participants were matched for age and physical activity, the females were shorter, had less mass, exhibited lower MVC and dynamic torque, slower angular velocity and less peak power than males. The females also displayed lower RTD, RVD, $\mathrm{RA}_{\text {sol }}$ and $\mathrm{RA}_{\mathrm{mg}}$ than males (Table 1).

Fatigue. Immediately following task termination, the fatigue-related change in MVC torque was not different for females than males $(p=0.38, d=0.47$, females post: $139.1 \pm 30.1 \mathrm{Nm}$, males post: 153.6 $\pm 26.9 \mathrm{Nm})$. The fatigue-related change in peak power was $\sim 15 \%$ less for females compared with males $(p=0.02, d=1.51$, Figure $1 \mathrm{~B})$, while the change in torque at peak power was $11 \%$ less $(p=0.03, d=1.51)$ with a trend for a smaller change in angular velocity at peak power $(p=0.08, d=1.38)$ for the females than males (Figure 1B). However, by task termination, there were no differences between sexes for percent change in RTD ( $p=0.11, d=1.08)$, RVD $(p=0.09$, $d=1.02), \mathrm{RA}_{\text {sol }}(p=0.75, d=0.20)$, or $\mathrm{RA}_{\mathrm{mg}}(p=0.68, d=0.18$, Figure 1B).

The fatigue-related change in peak power was positively and strongly correlated with a change in torque $\left(r=0.96, \mathrm{R}^{2}=0.92, p<0.001\right)$ and angular velocity $\left(r=0.91, \mathrm{R}^{2}=0.82, p<0.001\right)$, $\operatorname{RVD}\left(r=0.95, \mathrm{R}^{2}=0.90, p<0.001\right)$ and $\operatorname{RTD}\left(r=0.80, \mathrm{R}^{2}=0.63 p<0.001\right)$, but not $\mathrm{RA}_{\text {sol }}(r=0.51$, $\left.\mathrm{R}^{2}=0.26, p=0.45\right)$ nor $\mathrm{RA}_{\mathrm{mg}}\left(r=0.38, \mathrm{R}^{2}=0.14, p=0.15\right)$. 


\section{DISCUSSION}

The purpose of this study was to determine whether sex-related differences in performance fatigability of the plantar flexors exists following high-intensity shortening contractions. The main findings indicate that females were less fatigable than males, which was supported by less fatigue-related decrements in peak power, and torque and angular velocity $(p=0.08)$ at peak power for females than males (Figure 1B). The fatigue-related change in peak power was positively correlated with torque, velocity, RVD and RTD, but not rate of activation. Our current results indicate sex-related differences in performance fatigability may be related to mechanisms underlying time-dependent parameters within the muscle, but likely not those related to neuromuscular activation.

Although females were weaker, slower and exhibited lower rates of neuromuscular activation for the plantar flexors than males (Table 1), females were less fatigable, which is a different outcome than the limited number of reports on sex-related fatigue differences for maximal effort unconstrained shortening velocity tasks in other muscle groups (Senefeld et al. 2013, 2016). The differences may be owing to muscle fibre composition or activity patterns. Studies focusing on the elbow flexors and knee extensors suggest that both sexes demonstrate similar decrements in peak power (Senefeld et al. 2013, 2016). Yoon et al. (2015) reported that females were less fatigable than males during slow-velocity $(\sim 60 \%)$, low-resistance $(20 \% \mathrm{MVC})$ elbow flexions until task failure. Besides differences in angular velocity, the latter study (Yoon et al. 2015) used a larger work-to-rest ratio (lifting and lowering resistance over every $3 \mathrm{~s}$ ) than Senefeld et al. (2013, 2016), where subjects performed 90 maximal effort shortening contractions ( 1 contraction every 3 s). For our study, participants performed a total of 200 
shortening contractions every $\sim 1.5 \mathrm{~s}$. Thus, the differences in the present study and that of Senefeld et al. $(2013,2016)$ may be explained by the muscle tested, number of contractions, or the work-to-rest ratio of the respective tasks.

During maximal dynamic contractions, a critical component for achieving peak power is the capability to accelerate a limb quickly (Andersen et al. 2005). In the current study, females exhibited less fatigue-related decrements in torque and velocity compared with males, but no fatigue-related differences were detected for RTD, RVD and rate of activation between sexes. Additionally, fatigue-related changes in RTD and RVD were correlated with a change in peak power whereby the highest correlation was observed with RVD. Therefore, factors underlying RVD seem to be important for maintaining peak power during repetitive dynamic shortening contractions. Because rate of activation for both plantar flexors tested here were not correlated with fatigue-related changes in peak power, limitations within the muscle likely contributed to the sex-related differences of decrements in peak power.

Our study indicates that females are less fatigable than males when performing fast unconstrained velocity shortening plantar flexions and this sex-related difference is, at least partially, a result of time-dependent mechanical factors within the muscle that contribute to rapid power production. The sex-related differences in fatigability may only be revealed when the system is stressed with a large number of contractions - as shown here - or to task failure (Yoon et al. 2015). Future studies are required to determine the exact mechanisms and evaluate taskdependency affecting the sex-related differences in dynamic fatigability as it relates to peak power production and time-dependent paradigms. These results can be considered when designing exercise programs and may also lead to differences in work-related fatigue during dynamic strenuous activity for females and males. 


\section{ACKNOWLEDGEMENTS}

The authors thank the participants for volunteering in this study and Wendy Peters and Taylor Rios for their assistance with data analysis.

\section{CONFLICT OF INTEREST DISCLAIMER}

The authors report no conflicts of interest associated with this manuscript. 


\section{REFERENCES}

Andersen, L.L., Aagaard, P. 2006. Influence of maximal muscle strength and intrinsic muscle contractile properties on contractile rate of force development. Eur. J. Appl. Physiol. 96: 46-52. doi:10.1007/s00421-005-0070-z.

Andersen, L.L., Andersen, J.L., Magnusson, S.P., Suetta, C., Madsen, J.L., Christensen, L.R., Aagaard, P. 2005. Changes in the human muscle force-velocity relationship in response to resistance training and subsequent detraining. J. Appl. Physiol. 99: 87-94. doi:10.1152/japplphysiol.00091.2005.

Cheng, A.J., Rice, C.L. 2005. Fatigue and recovery of power and isometric torque following isotonic knee extensions. J. Appl. Physiol. 99: 1446-1452. doi:10.1152/japplphysiol.00452.2005.

Dalton, B.H., Allen, M.D., Power, G.A., Vandervoort, A.A., Rice, C.L. 2014. The effect of knee joint angle on plantar fl exor power in young and old men. Exp. Gerontol. 52: 70-76. doi:10.1016/j.exger.2014.01.011.

Dalton, B.H., Power, G.A., Allen, M.D., Vandervoort, A.A., Rice, C.L. 2013. The genu effect on plantar flexor power. Eur. J. Appl. Physiol. 113: 1431-1439. doi:10.1007/s00421-012-25600 .

Dalton, B.H., Power, G.A., Paturel, J.R., Rice, C.L. 2015. Older men are more fatigable than young when matched for maximal power and knee extension angular velocity is unconstrained. Age, 37: 49. doi:10.1007/s11357-015-9790-0.

Dalton, B.H., Power, G.A., Vandervoort, A.A., Rice, C.L. 2012. The age-related slowing of voluntary shortening velocity exacerbates power loss during repeated fast knee extensions. Exp. Gerontol. 47: 85-92. doi:10.1016/j.exger.2011.10.010.

Dalton, B.H., Power, G.A., Vandervoort, A.A., Rice, C.L., Dalton, B.H., Power, G.A., Vandervoort, A.A., Rice, C.L. 2010. Power loss is greater in old men than young men during fast plantar flexion contractions. J. Appl. Physiol. 109: 1441-1447. doi:10.1152/japplphysiol.00335.2010.

Hunter, S.K. 2016. Sex differences in fatigability of dynamic contractions. Exp. Physiol. 101: 250-255. doi:10.1113/EP085370.

Labarbera, K.E., Murphy, B.G., Laroche, D.P., Cook, S.B. 2013. Sex differences in blood flow restricted isotonic knee extensions to fatigue. J. Sports Med. Phys. Fitness, 53: 444-452. PMID:23828293.

Maughan, R.J., Harmon, M., Leiper, J.B., Sale, D., Delman, A. 1986. Endurance capacity of untrained males and females in isometric and dynamic muscular contractions. Eur. J. Appl. Physiol. 55: 395-400. doi:10.1007/BF00422739.

Pincivero, D.M., Coelho, A.J., Campy, R.M. 2004. Gender differences in perceived exertion during fatiguing knee extensions. Med. Sci. Sports Exerc. 36: 109-117. doi:10.1249/01.MSS.0000106183.23941.54.

Power, G.A., Dalton, B.H., Rice, C.L., Vandervoort, A.A. 2010. Delayed recovery of velocitydependent power loss following eccentric actions of the ankle dorsiflexors. J. Appl. Physiol. 109: 669-676. doi:10.1152/japplphysiol.01254.2009.

Power, G.A., Dalton, B.H., Rice, C.L., Vandervoort, A.A. 2011. Reproducibility of velocitydependent power: before and after lengthening contractions. Appl. Physiol. Nutr. Metab. 36: 626-33. doi:10.1139/h11-068.

Power, G.A., Dalton, B.H., Rice, C.L, Vandervoort, A.A. 2012. Power loss is greater following 
lengthening contractions in old versus young women. Age, 34: 737-750. doi:10.1007/s11357-011-9263-z.

Senefeld, J., Yoon, T., Bement, M.H., Hunter, S.K. 2013. Fatigue and recovery from dynamic contractions in men and women differ for arm and leg muscles. Muscle Nerve, 48: 436439. doi:10.1002/mus.23836.

Senefeld, J., Yoon, T., Hunter, S.K. 2016. Age differences in dynamic fatigability and variability of arm and leg muscles: Associations with physical function. Exp. Gerontol. 87: 74-83. doi:10.1016/j.exger.2016.10.008.

Wallace, J.W., Power, G.A., Rice, C.L., Dalton, B.H. 2016. Time-dependent neuromuscular parameters in the plantar flexors support greater fatigability of old compared with younger males. Exp. Gerontol. 74: 13-20. doi:10.1016/j.exger.2015.12.001.

Yoon, T., Doyel, R., Widule, C., Hunter, S.K. 2015. Sex differences with aging in the fatigability of dynamic contractions. Exp. Gerontol. 70: 1-10. doi:10.1016/j.exger.2015.07.001. 
Table 1. Participant characteristics and pre-fatigue neuromuscular properties of the plantar flexors.

\begin{tabular}{llllll}
\hline & Female & Male & \%diff & $\boldsymbol{p}$-value & $\boldsymbol{d}$ \\
Age (years) & $21.9 \pm 2.5$ & $22.9 \pm 2.5$ & - & 0.444 & 0.40 \\
Height (cm) & $169.3 \pm 5.1^{*}$ & $177.5 \pm 3.9$ & 5 & 0.002 & 1.60 \\
Weight (kg) & $63.7 \pm 7.5^{*}$ & $73.6 \pm 6.6$ & 6 & 0.012 & 1.32 \\
Physical Activity (h/week) & $8.9 \pm 4.1$ & $9.8 \pm 4.3$ & - & 0.699 & 0.20 \\
MVC (Nm) & $163.2 \pm 28.9 \dagger$ & $189.2 \pm 22.2$ & 16 & 0.057 & 0.90 \\
Power (W) & $127.9 \pm 36.6^{*}$ & $183.0 \pm 28.9$ & 43 & 0.004 & 1.50 \\
Torque (Nm) & $61.1 \pm 11.0^{*}$ & $81.6 \pm 12.2$ & 34 & 0.002 & 1.86 \\
Velocity (rad/s) & $2.0 \pm 0.2^{*}$ & $2.2 \pm 0.1$ & 11 & 0.007 & 1.31 \\
RTD (Nm/s) & $632.2 \pm 137.2^{*}$ & $850.7 \pm 160.3$ & 35 & 0.008 & 1.59 \\
RVD (rad/s $\mathbf{2})$ & $36.5 \pm 5.6^{*}$ & $45.1 \pm 4.2$ & 24 & 0.003 & 1.55 \\
RA $\mathbf{s o l}(\mathbf{m V} / \mathbf{s})$ & $0.9 \pm 0.4^{*}$ & $1.5 \pm 0.6$ & 72 & 0.035 & 1.62 \\
$\mathbf{R A}_{\mathbf{m g}}(\mathbf{m V} / \mathbf{s})$ & $1.2 \pm 0.4 \dagger$ & $1.8 \pm 0.7$ & 50 & 0.066 & 1.26 \\
\hline
\end{tabular}

The females were shorter, lighter, weaker, slower, less powerful and displayed a lower rate of activation than the males, but were matched for age and physical activity levels. Maximal voluntary isometric contraction (MVC), peak power (Power), torque at peak power (Torque), velocity at peak power (Velocity), peak dynamic rate of torque development (RTD), peak rate of velocity development (RVD), peak rate of activation for the soleus $\left(\mathrm{RA}_{\mathrm{sol}}\right)$ and medial gastrocnemius $\left(\mathrm{RA}_{\mathrm{mg}}\right)$, percent sex-related difference (\%diff), and Cohen's $d$ value $(d)$. Values are means \pm standard deviations. * represents $p$ $<0.05$ and $\uparrow$ represents $p<0.08$. 


\section{FIGURE LEGEND}

Figure 1. Panel A. Representative data from one female participant. The data includes angular velocity, torque and medial gastrocnemius electromyography (EMG) for two dynamic shortening contractions at the beginning (within the first five contractions of the fatigue task) and end (within the last five contractions of the fatigue task). Panel B. Fatigue-related percent change values in maximal voluntary isometric contraction (MVC), peak power, torque and angular velocity at peak power, rate of torque development (RTD), rate of velocity development (RVD), and soleus ( $\mathrm{RA}_{\text {sol }}$ ) and medial gastrocnemius $\left(\mathrm{RA}_{\mathrm{mg}}\right.$ ) rate of activation for females (filled) and males (unfilled). The females exhibited less change in peak power, torque and velocity, but similar changes in MVC, RTD, RVD, $\mathrm{RA}_{\text {sol }}$ and $\mathrm{RA}_{\mathrm{mg}}$. Values are means \pm standard deviations. ${ }^{*}$ depicts difference between sexes $(p<0.05)$ and $\dagger$ represents a trend $(p<0.08)$. 
A

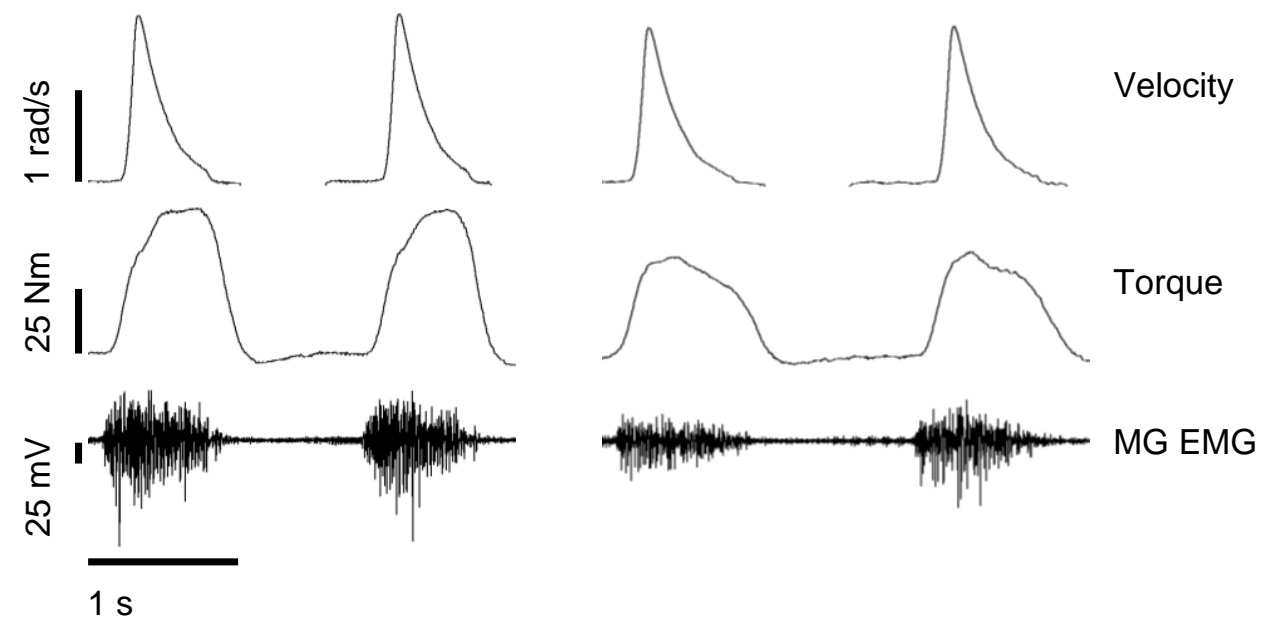

B

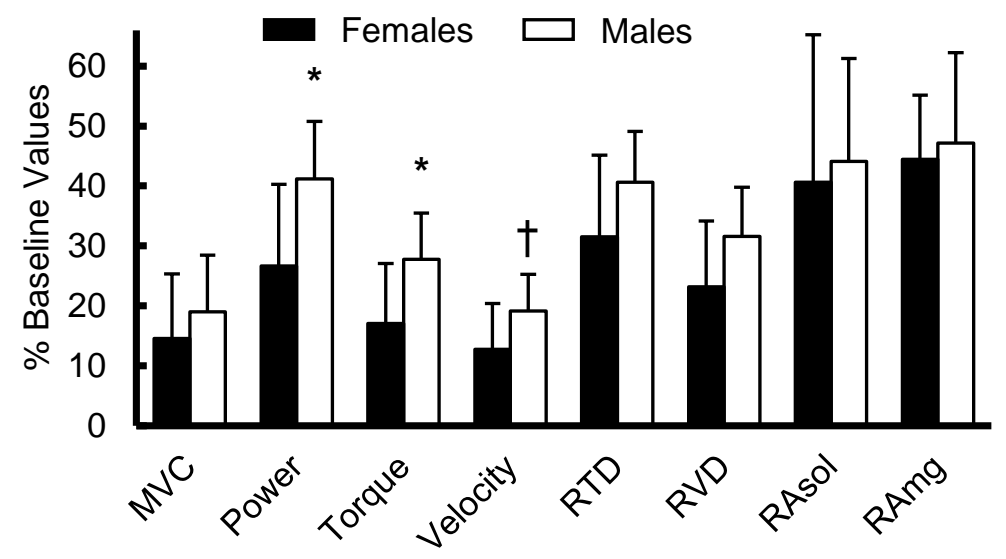

